\title{
MODERNIZAÇÃO DO CONTROLE DOS ELETRODOS DA ACIARIA ELÉTRICA DA CSN AÇOS LONGOS*
}

\author{
Silvio de Carvalho Sabença ${ }^{1}$ \\ Cristiano Garcia Gomes ${ }^{2}$ \\ Wallace Marcelino Rocha ${ }^{3}$ \\ Marcus Daniel Almada 4
}

\section{Resumo}

Um forno a arco elétrico é um tipo de forno industrial que usa uma corrente elétrica alternada para produzir calor. Estes equipamentos servem como uma alternativa para os altos fornos tradicionais, e desempenham um papel importante na produção de aço. Em um forno de arco elétrico, a corrente elétrica passa através dos eletrodos de grafite, do aço e de outros metais, o que resulta na produção de aço de modo mais rápido e eficiente. A queda de tensão no arco é, praticamente, proporcional à distância entre o eletrodo e a carga. O eletrodo corresponde ao polo positivo (+) e a carga o negativo (-). Em corrente alternada os pólos, positivo e negativo, trocam de posição a cada meio ciclo da tensão de rede. Sob condições de arco, forma-se, no eletrodo e na carga, uma finíssima camada altamente ionizada devida a uma altíssima concentração do campo elétrico nestas zonas. No forno o arco se extingue quando a corrente se anula e reacende, quando a tensão volta a crescer. Devido a constantes quebra de eletrodo foi montado o grupo de trabalho com objetivo de reduzir o consumo dos eletrodos, otimização do consumo de energia, redução do Power On, aumento da potência média e redução nas quebras de eletrodos. Esta implantação foi extremamente importante pois o mesmo está garantindo a continuidade operacional, de forma segura e confiável, de um dos equipamentos mais importantes para a produção de aço na Usina.

Palavras-chave: Aciaria elétrica; Forno elétrico à arco; Controle de eletrodos.

\section{MODERNIZATION OF ELECTRODE CONTROL OF STEEL MAKING - CSN LONG}

\section{STEEL}

\section{Abstract}

An electric arc furnace is a kind of industrial furnace using an alternate current to produce heat. These devices serve as an alternative to the traditional Blast Furnace, and play an important role in the production of steel. In an electric arc furnace, the electrical current passes through the graphite electrodes, steel and other metals, which results in more efficient and faster steel production. The electrode corresponds to the positive pole (+) and scrap load to the negative pole (-). In AC, both poles change position at every half cycle of the line voltage. Under normal arc conditions it is formed a highly ionized thin layer at the electrode tip and the load, a thin highly ionized layer due to a high concentration of electric field in these areas. In the furnace the arc is extinguished when the current is zero and reignites when the voltage increases again. Due to constant electrode breakage, a team work was formed in order to reduce the consumption of the electrodes, optimization of energy consumption, reduction of PowerOn time, increased average power and reduction in electrode breakage. This project was extremely important because it has ensured better continuous operation, higher safety and reliability at one of the most important equipment for steel production in the plant.

Keywords: Steel making; Electric arc furnace; Electrode control.

1 Engenheiro Eletrônico, Pós-Graduação, Engenheiro Especialista de Engenharia, GGOS/GEM, CSN, Volta Redonda, RJ, Brasil.

2 Engenheiro de Automação, Graduado, Engenheiro Especialista de Manutenção, GGNP/GNM, CSN, Volta Redonda, RJ, Brasil.

3 Engenheiro de Automação, Graduado, Engenheiro Pleno de Manutenção, GGNP/GNM, CSN, Volta Redonda, RJ, Brasil.

4 Engenheiro Eletricista, Graduado, Engenheiro Especialista de Manutenção, GGCE/GDE, CSN, Volta Redonda, RJ, Brasil. 


\section{INTRODUÇÃO}

Para compreender a operação de um forno a arco é necessário entender bem a natureza do arco. $\mathrm{O}$ arco é gerado pela passagem da corrente elétrica através de uma atmosfera altamente ionizada. A queda de tensão no arco é, praticamente, proporcional à distância entre o eletrodo e a carga. O eletrodo corresponde ao polo positivo (+) e a carga o negativo (-). Em corrente alternada os polos, positivo e negativo, trocam de posição a cada meio ciclo da tensão de rede. Sob condições de arco, forma-se, no eletrodo e na carga, uma finíssima camada altamente ionizada devida a uma altíssima concentração do campo elétrico nestas zonas. No forno o arco se extingue quando a corrente se anula e reacende, quando a tensão volta a crescer. Isto ocorre a cada meio ciclo. $\mathrm{O}$ arco reacende porque todo o espaço entre os polos permanece ionizado durante a inversão da polaridade.

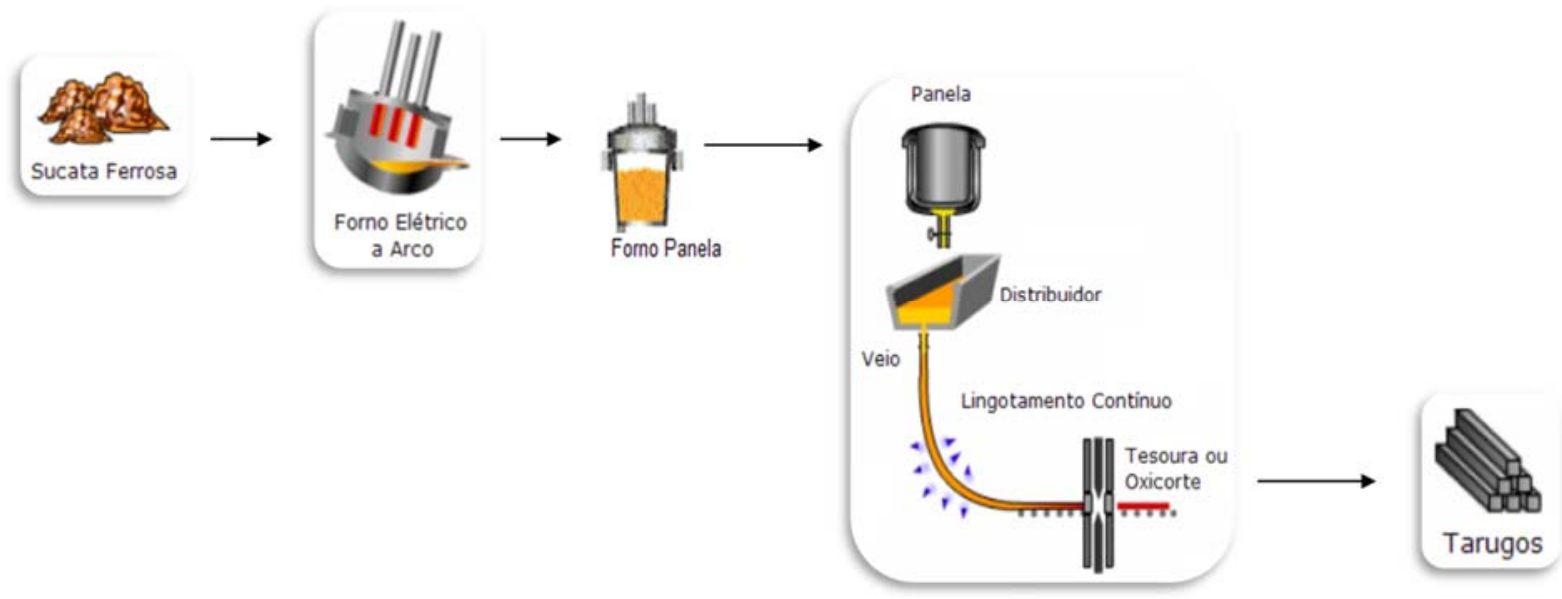

Figura 1 - Aciaria da CSN LONGOS

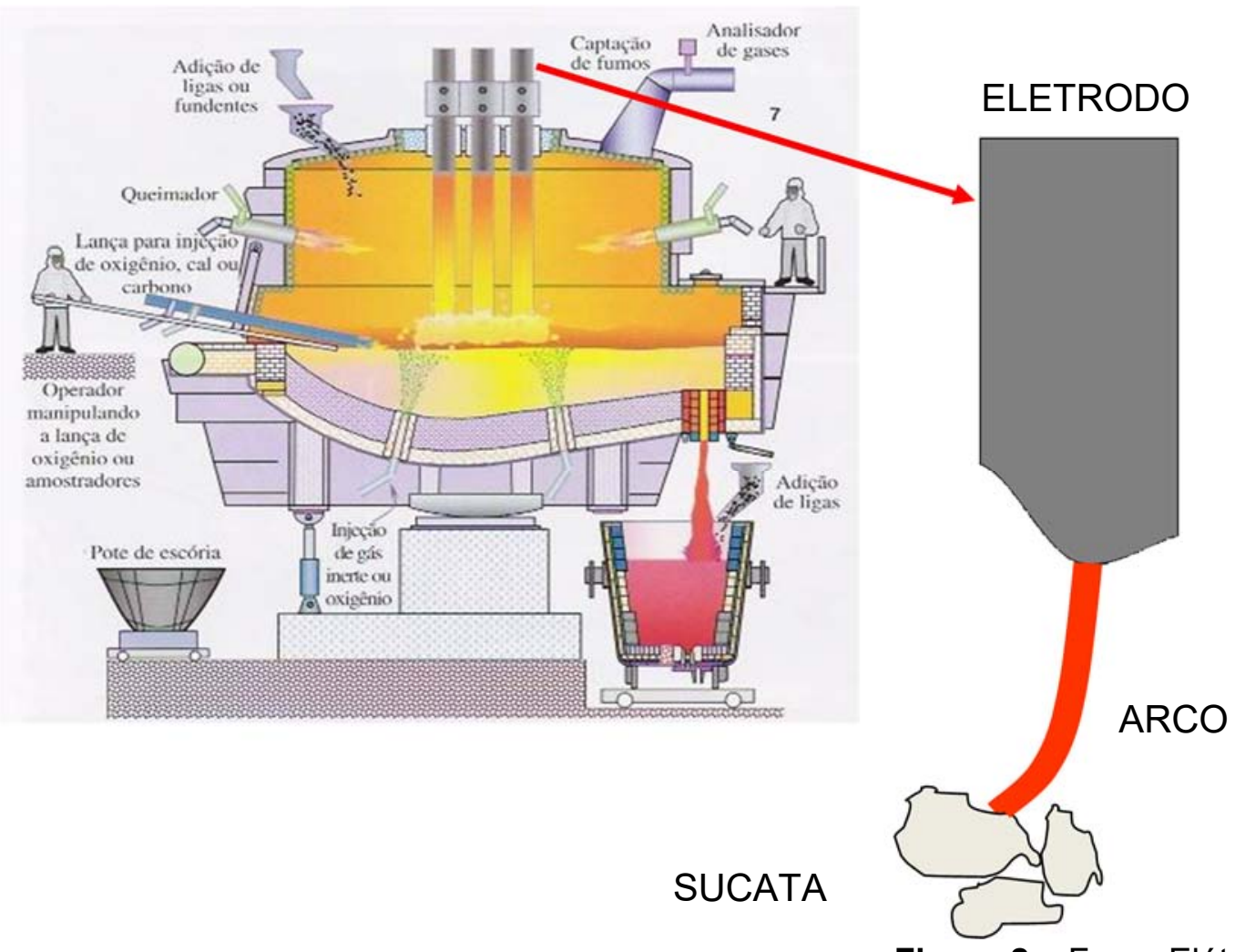

Figura 2 - Forno Elétrico a Arco 


\subsection{ARCO ELÉTRICO COMO CONDUTOR}

- Flexível

- Movimenta-se de acordo com as forças eletromagnéticas

- A posição do eixo do arco e seu comprimento determinam a direção do fluxo de calor

- Volume do arco depende:

- Tensão (comprimento)

- Corrente (diâmetro)

- Condições de transferência de calor dependem da tensão e corrente

\subsection{FORNO DE ARCO ELÉTRICO DA CSN - AÇOS LONGOS}

- Fabricante $=$ CISDI - CHINA

- Diâmetro carcaça $=4800 \mathrm{~mm}$ livre

- Diâmetro eletrodos = 20"

- Circulo primitivo eletrodos $=1000 \mathrm{~mm}$

- Distância lateral eletrodos até parede $=164 \mathrm{~cm}$

- Reatância sistema secundário (Estimado) $=2,5 \mathrm{~m} \Omega$

- Resistência sistema secundário (Estimado) $=0,5 \mathrm{~m} \Omega$

- Sistema de regulação de eletrodos = Simatic \$7-300 e Válvulas proporcionais

Rexroth

- Potência Nominal = 45 MVA

- Tensão Primária $=34,5$ kV

\subsection{O REGULADOR DE ELETRODO}

Para manter o arco aceso e, tanto quanto possível, com a tensão e a corrente desejadas, são utilizados os reguladores de posição dos eletrodos. Em geral, o que se deseja é manter constante a corrente de arco, no valor ótimo de projeto do forno, para as diversas tensões secundárias do transformador de alimentação do forno. Estes valores de corrente e tensão são estabelecidos pela operação e o regulador de arco deverá posicionar os eletrodos de modo a mantê-los constantes, automaticamente. O regulador movimenta cada eletrodo, de modo independente, através do sinal de erro decorrente da comparação entre um sinal proporcional à corrente de carga e outro proporcional à tensão de fase do arco. Estas tensões e correntes são medidas no barramento secundário do transformador do forno. $\mathrm{O}$ ideal seria medir diretamente a tensão de arco, porém, isto é praticamente impossível, pois não se pode atingir a ponta dos eletrodos. Quando o arco muda de posição, buscando uma peça metálica mais distante, o comprimento e a tensão do arco aumentam e a corrente diminui.

O regulador digital de eletrodos baseia-se no sistema de impedância constante e corrente constante, podendo ser aplicado para movimentação de eletrodos com motores CA e CC, e sistemas hidráulicos com servoválvulas ou válvulas proporcionais.

As funções principais de um regulador de eletrodos são:

- Ajuste rápido do arco durante as condições normais de operação, evitando-se tempos prolongados de curto circuito e conseqüente desligamento por sobrecorrente;

- Manter a corrente de setpoint dentro dos valores ajustados;

- Reduzir distúrbios na rede;

- Flexibilidade operacional. 


\subsection{DIAGRAMA DE REGULAÇÃO}

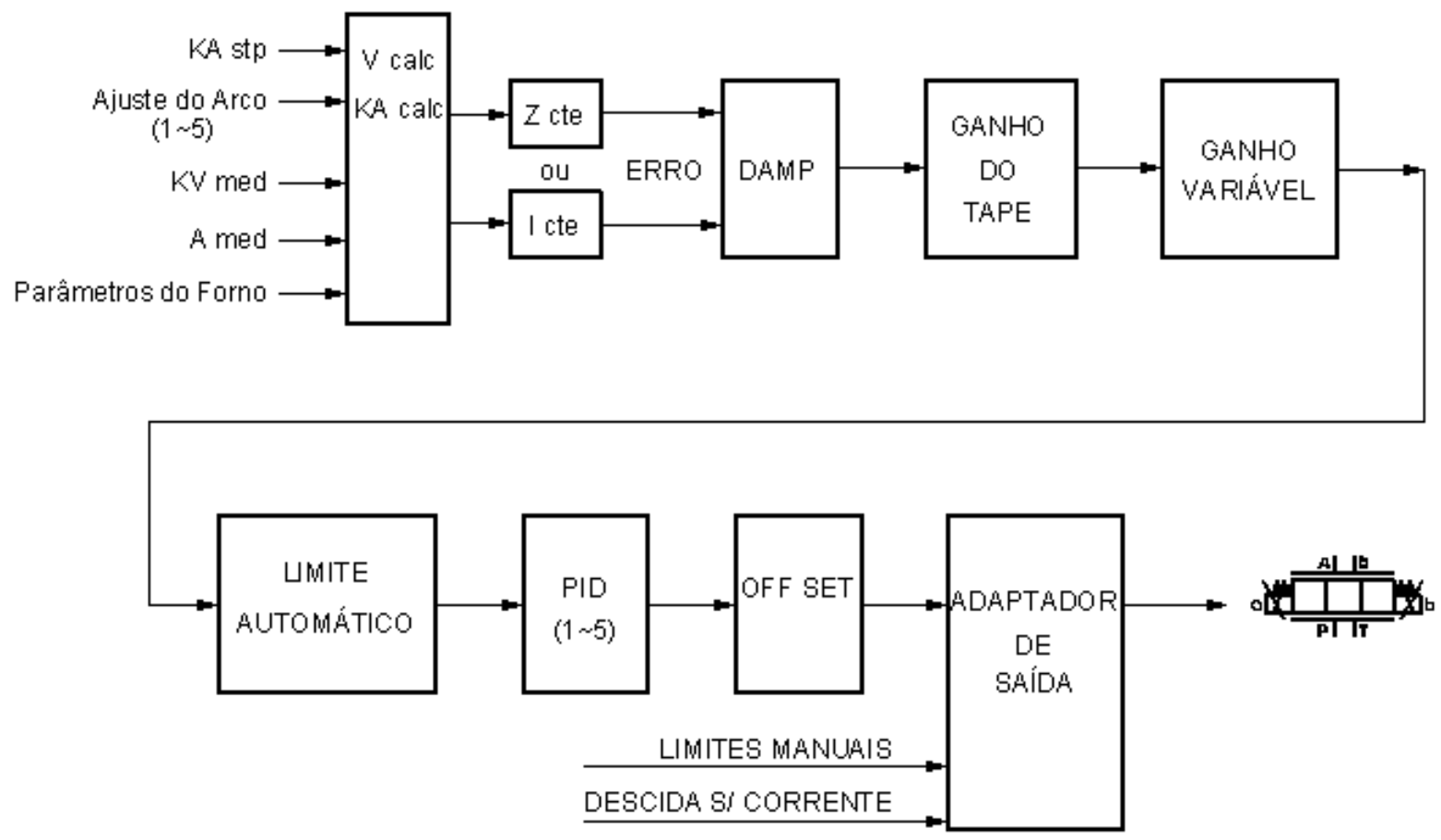

Figura 3 - Diagrama de controle

\section{PROBLEMAS COM O SISTEMA DE CONTROLE DE ELETRODOS}

2.1. Sistema original não tem sistema anti-quebra de eletrodos;

2.2. Elevado consumo de energia;

2.3. Sistema original não possibilita alteração de parâmetros (caixa preta);

2.4. Dificuldade de manutenção: sistemas sem comentários e blocos de programação fechados;

Com os principais problemas acima acarreta um elevado número de quebras de eletrodos, 134 eletrodos quebrados no período de 5 meses aumentando o POWER ON médio para 55 minutos. 


\section{Uma Corrida Normal}

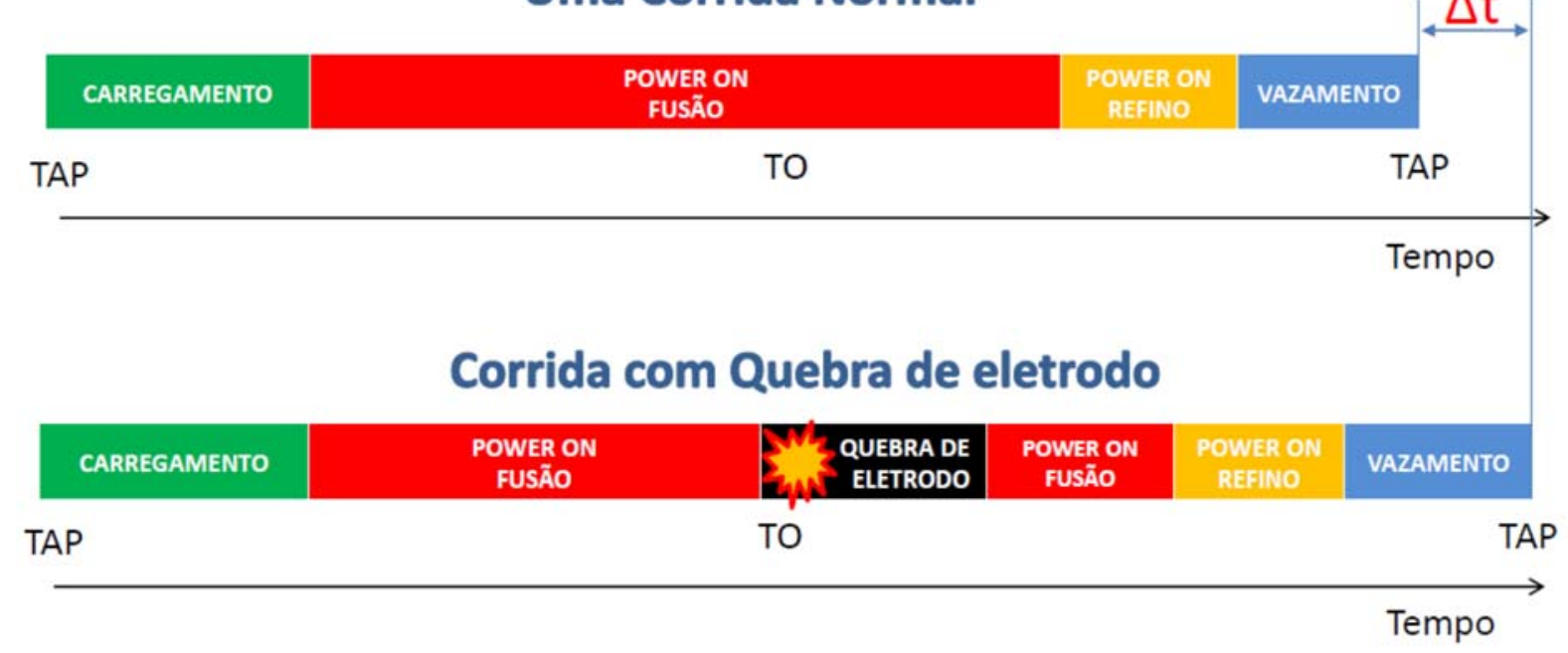

Figura 4 - Produção Tap-To-Tap

2.5. Diagrama de Regulação dos Eletrodos Original

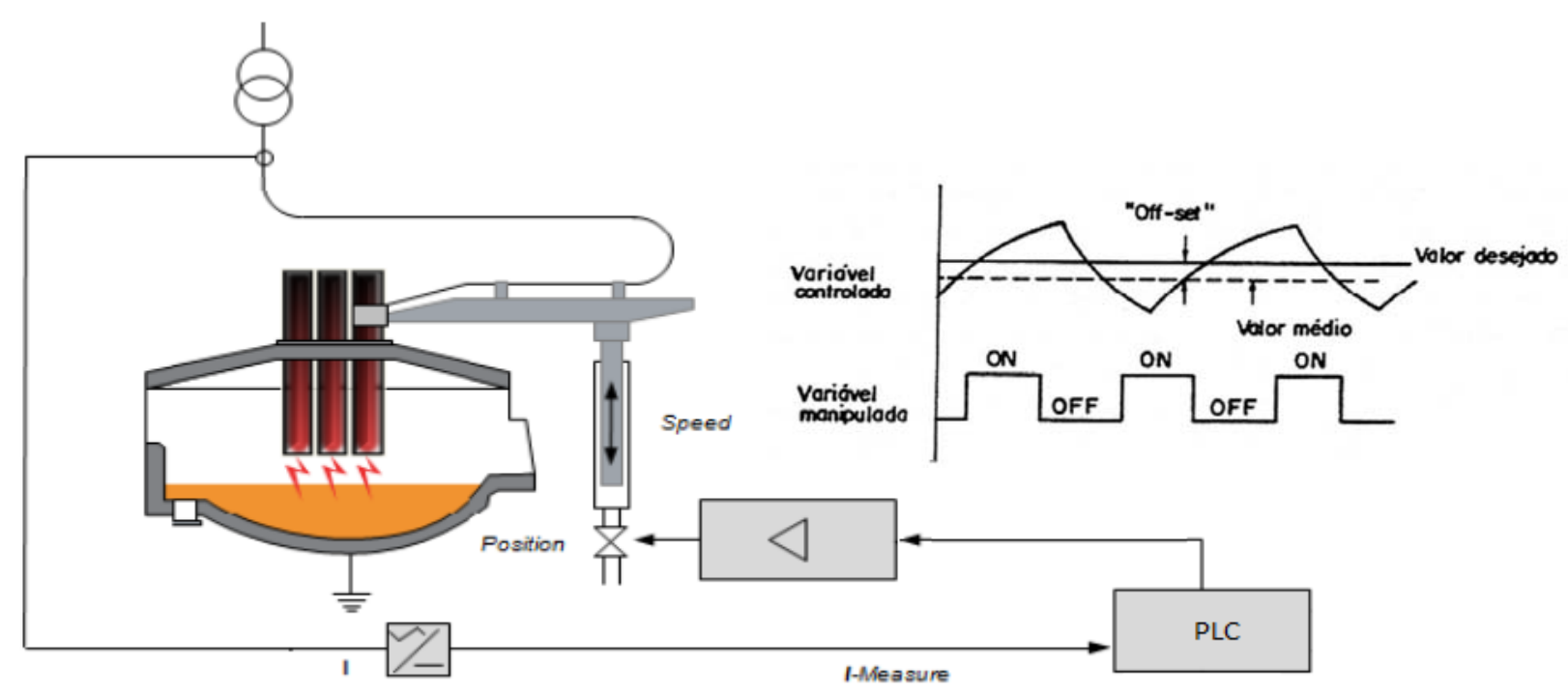

Figura 5 - Controle Original

O controle Original do Regulador dos Eletrodos é tipo On-Off, não tendo controle proporcional, acarretando mudanças de posições abruptas ocasionando quebra de eletrodos. 
3. PLANEJAMENTO PARA NORMALIZAÇÃO DO CONTROLE DOS ELETRODOS

3.1.1. Estudo do sistema de regulação do eletrodo original

3.1.2. Elaboração de especificação técnica novo sistema

3.1.3. Aquisição de equipamentos

3.1.4. Instalação do novo sistema

3.1.5. Integração com a automação existente

3.1.6. Comissionamento

3.1.7. Start Up

\subsection{CRONOGRAMA MACRO}

\section{Ação: Análise}

\section{Prazo: Março/14}

\section{Responsável : Grupo}

\section{Ação: Especificação}

Prazo: Abril/14

Responsável : Grupo

Ação: Aquisição

Prazo: Agosto/14

\section{Responsável : Grupo}

\section{Ação: StartUp}

Prazo: Setembro/14

Responsável : Grupo

\subsection{ESPECIFICAÇÃO TÉCNICA DO NOVO SISTEMA}

3.3.1. Sistema Anti-quebra de Eletrodo

3.3.2. Disponibilizar sistema HMI

3.3.3. Sistema aberto possibilitando modificações pela manutenção

3.3.4. Possibilidade de alteração de parâmetros de processo

3.3.5. Instalar nova medição de corrente e tensão direta no secundário

3.3.6. Manter o sistema de comando eletro-hidráulico dos eletrodos

3.3.7. Possibilitar o chaveamento para o sistema antigo sem atrasos se necessário 


\section{NOVO SISTEMA DE CONTROLE DOS ELETRODOS}

\subsection{ARQUITETURA DE AUTOMAÇÃO - Sistema Original x Sistema Novo}

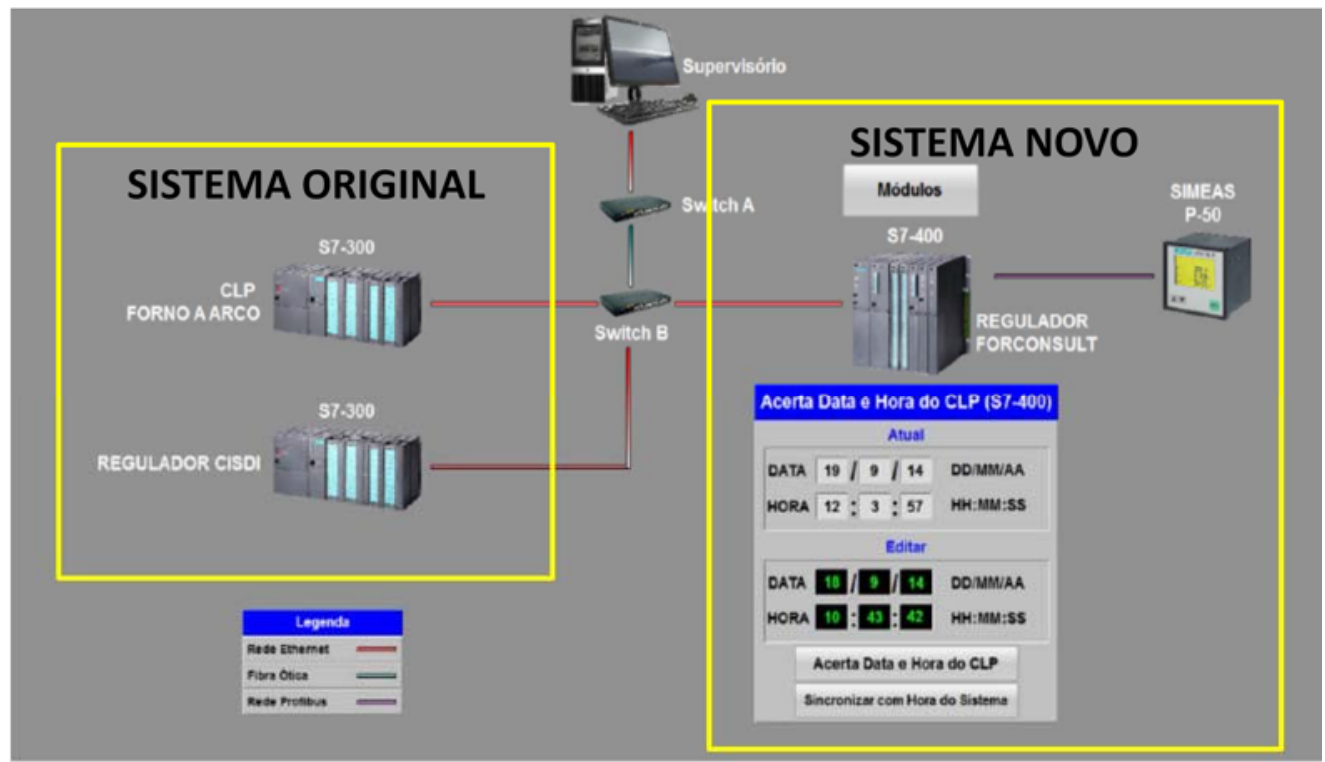

Figura 6 - Arquitetura de Automação O sistema original foi mantido e pode ser utilizado como standby.

\subsection{ATUAÇÃO DO SISTEMA ANTI-QUEBRA DE ELETRODOS}

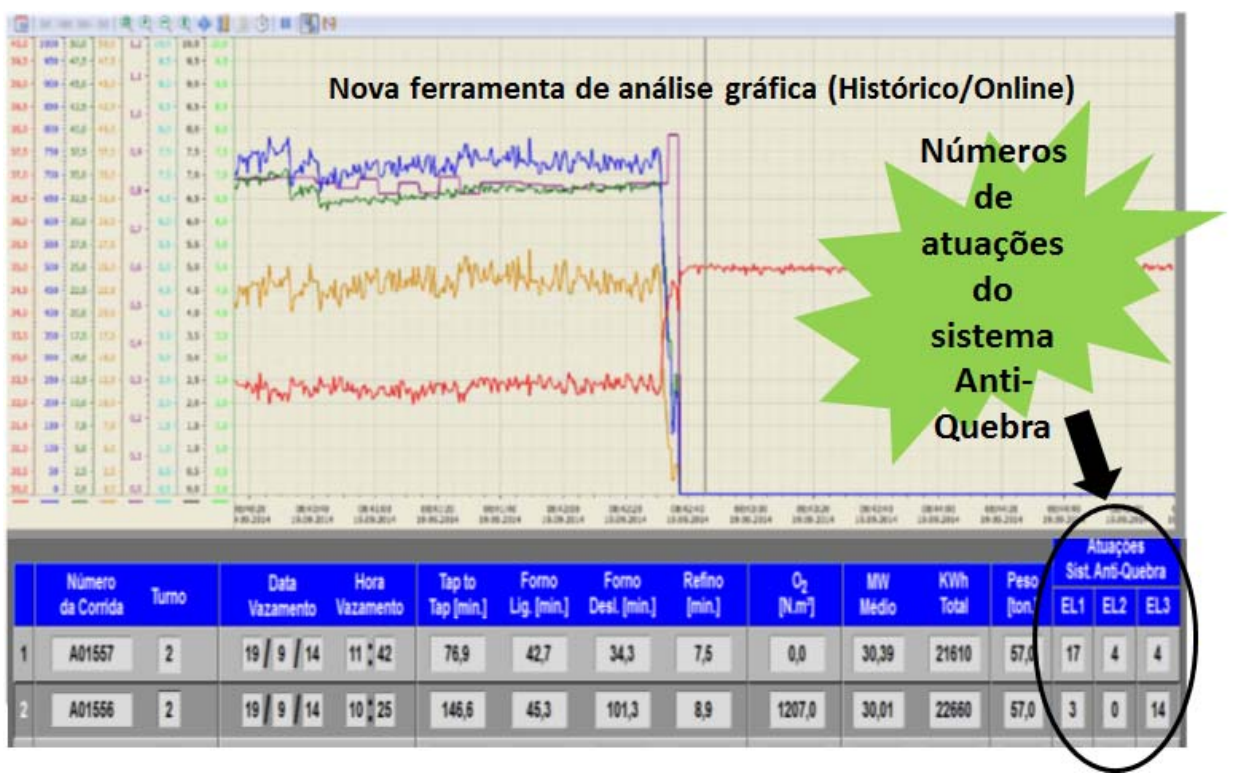

Figura 7 - Sistema Anti-quebra 


\subsection{NOVAS TELAS DE CONTROLE DE OPERAÇÃO}

4.4.

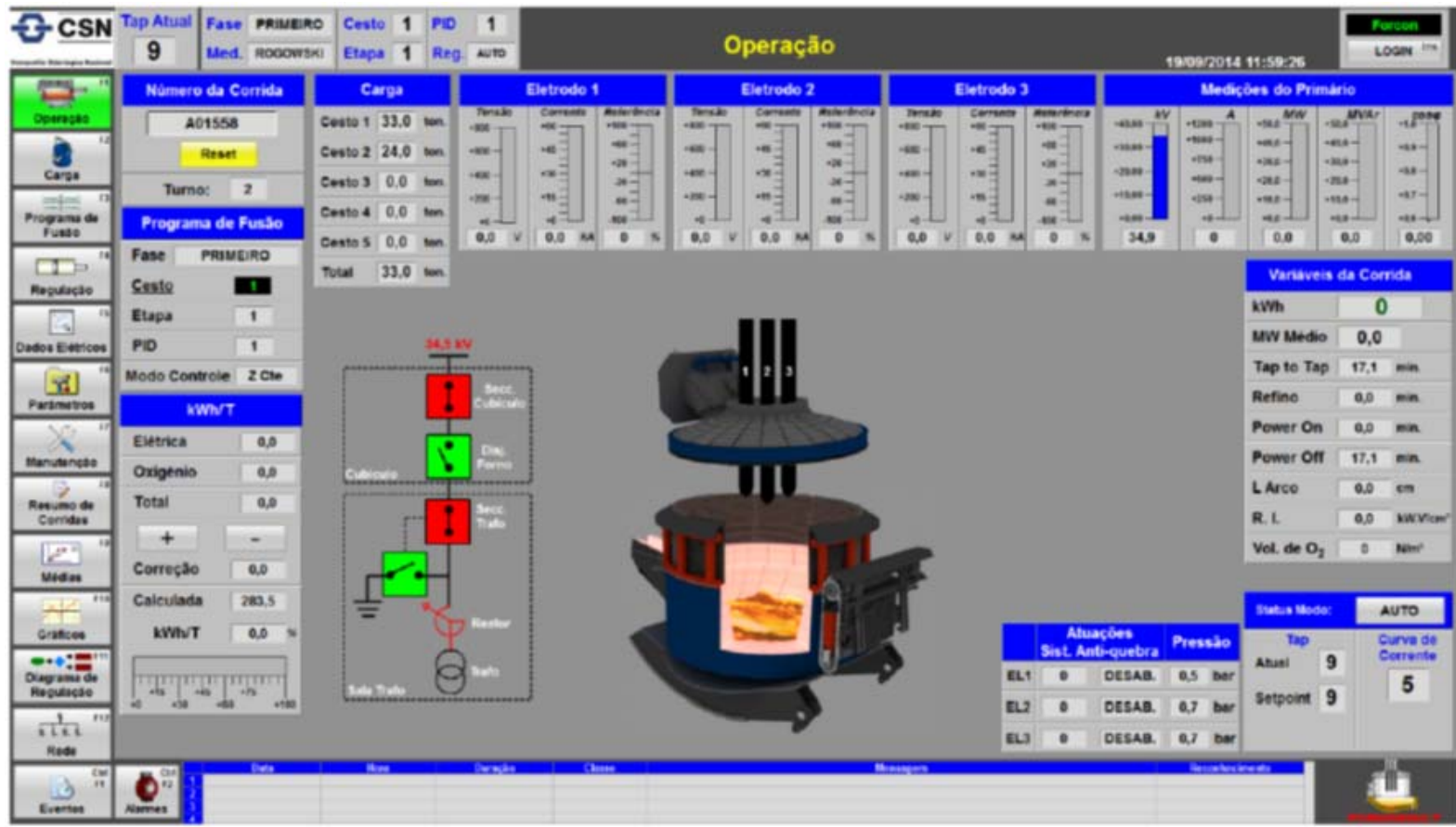

Figura 8 - Tela de Processo

\subsection{TELA DE MALHAS DE CONTROLE DOS ELETRODOS}

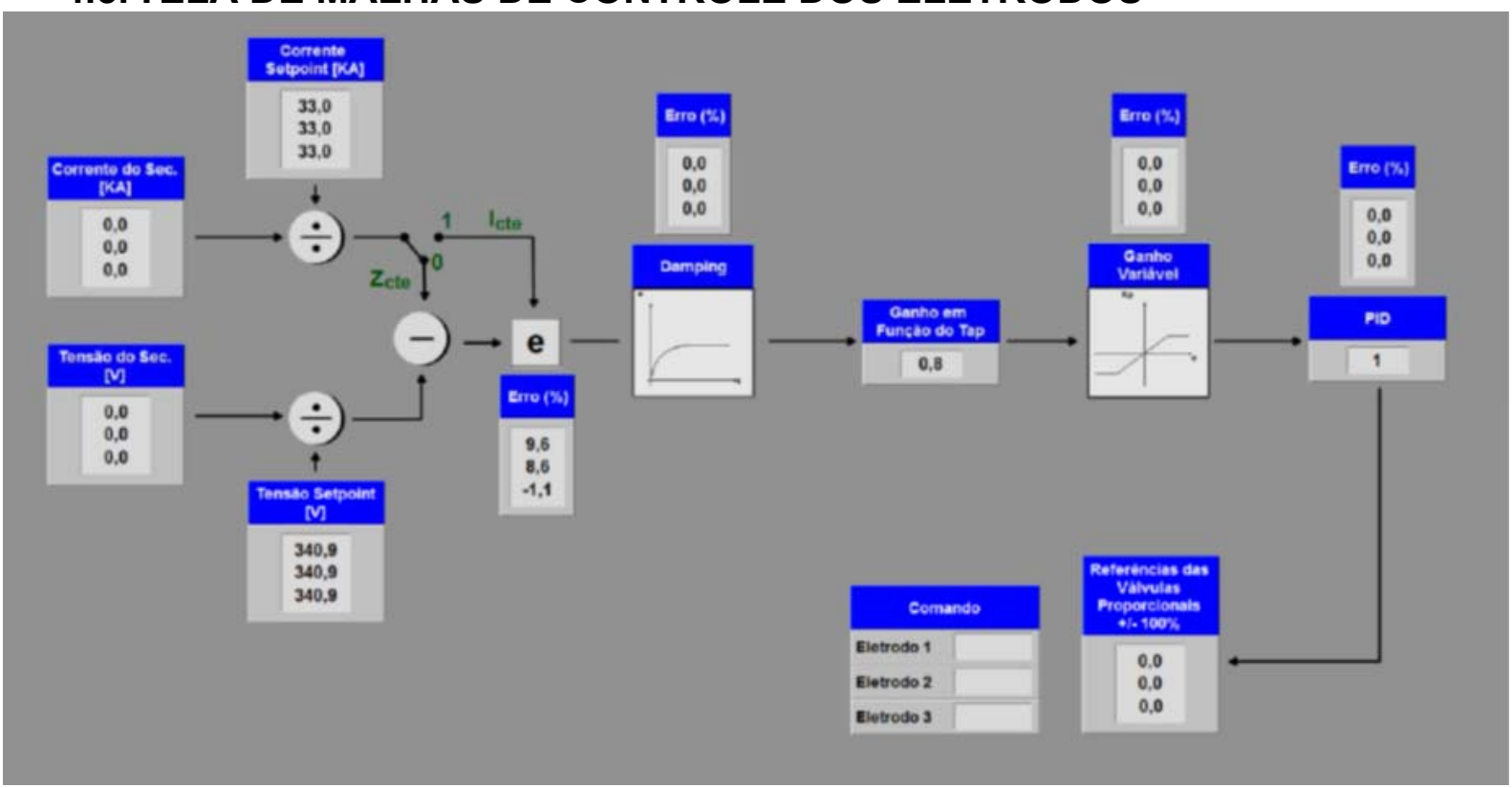

Figura 9 - Tela de Controle 


\subsection{VERIFICAÇÃO DOS RESULTADOS ENTRE CONTROLE ORIGINAL E O NOVO SISTEMA}

\subsubsection{Melhorias com o Novo Sistema}

4.6.1.1. Sistema anti-quebra dos eletrodos utilizando a pressão individual das colunas dos eletrodos;

4.6.1.2. Melhor tempo de resposta na regulação dos eletrodos (alteração do controle de On-Off para Proporcional);

4.6.1.3. Melhor estabilidade do arco e consequente maior potência média 4.6.1.4. Regulação mais suave na descida e carga dos eletrodos.

\subsubsection{Benefícios com Novo Sistema}

4.6.2.1. Redução nas paradas por quebras de eletrodos

4.6.2.2. Redução do Consumo dos Eletrodos

4.6.2.3. Redução do Descarte de Eletrodos de Grafite

4.6.2.4. Otimização do Consumo de energia

4.6.2.5. Redução do tempo de Power ON

4.6.2.6. Estabilidade de Potência (menor variação)

4.6.2.7. Maior potência média

\subsubsection{Melhoria da Média Mensal do Tempo de POWER ON}

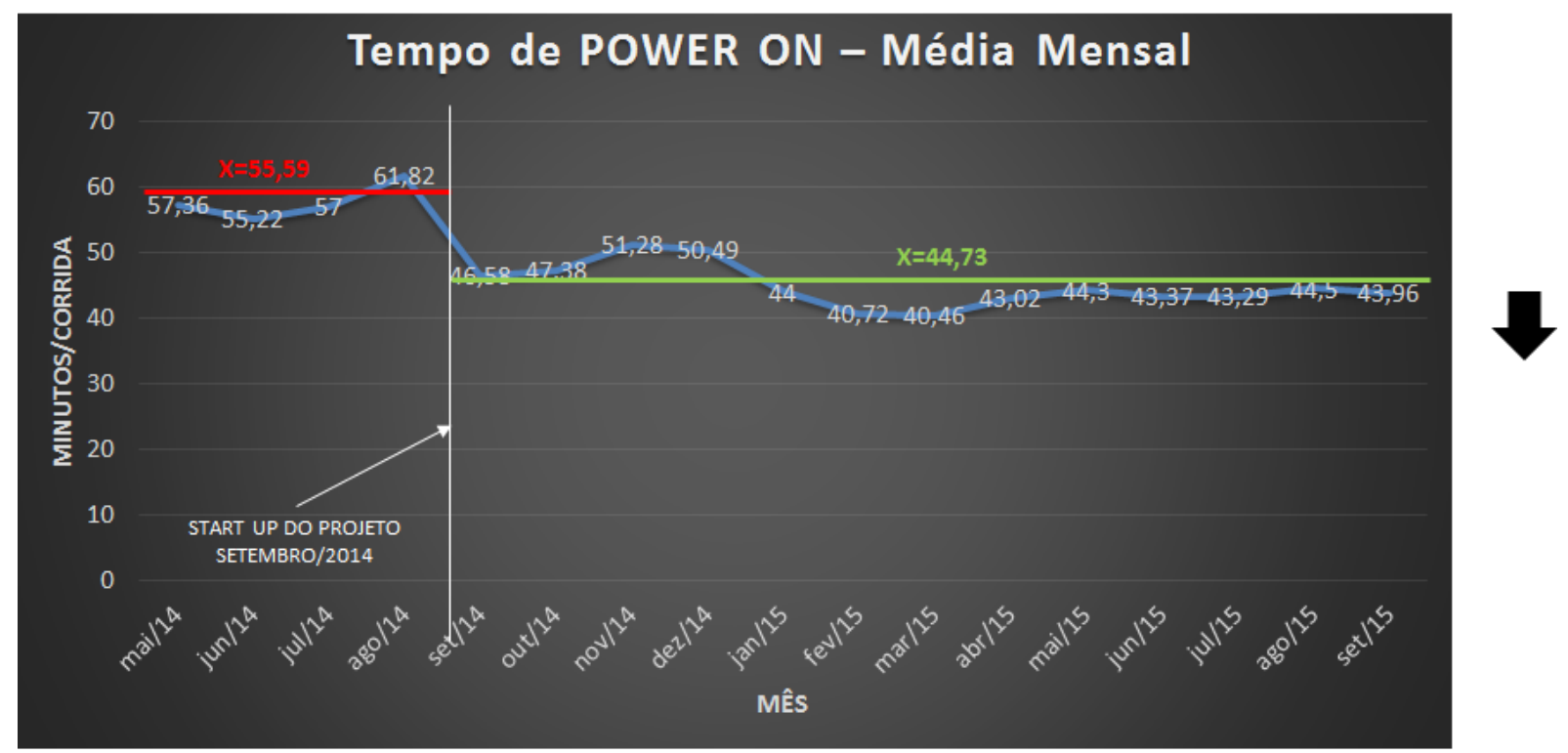

Fonte: ROLAP E PROCOM

Figura 10 - Tempo de POWER ON 


\subsubsection{Aumento da Produção de Aço Liquido}

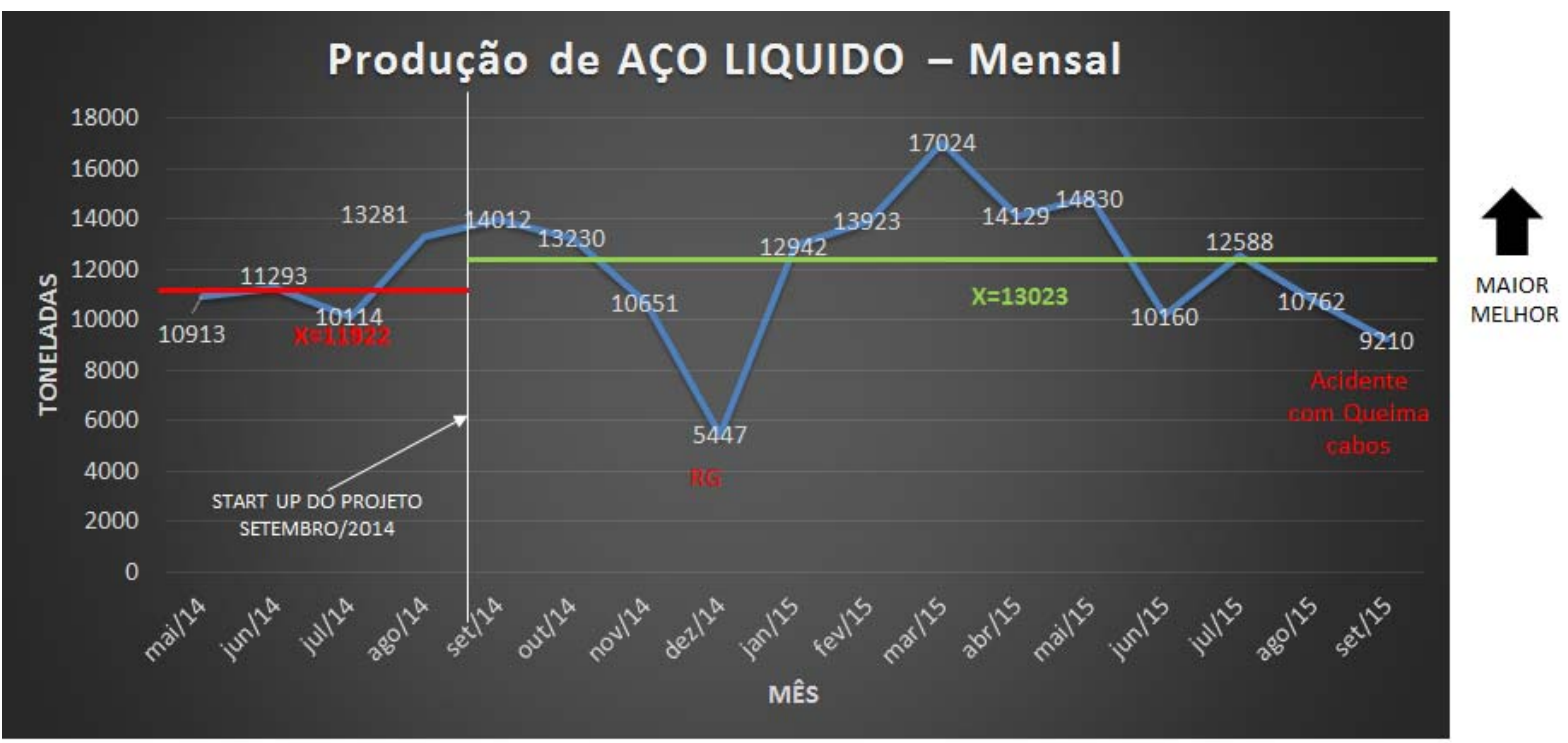

FONTE: ROLAP E PROCOM

Figura 11 - Produção

\subsubsection{Redução da Quebra de Eletrodos}

\section{Média de Quebra de Eletrodos}

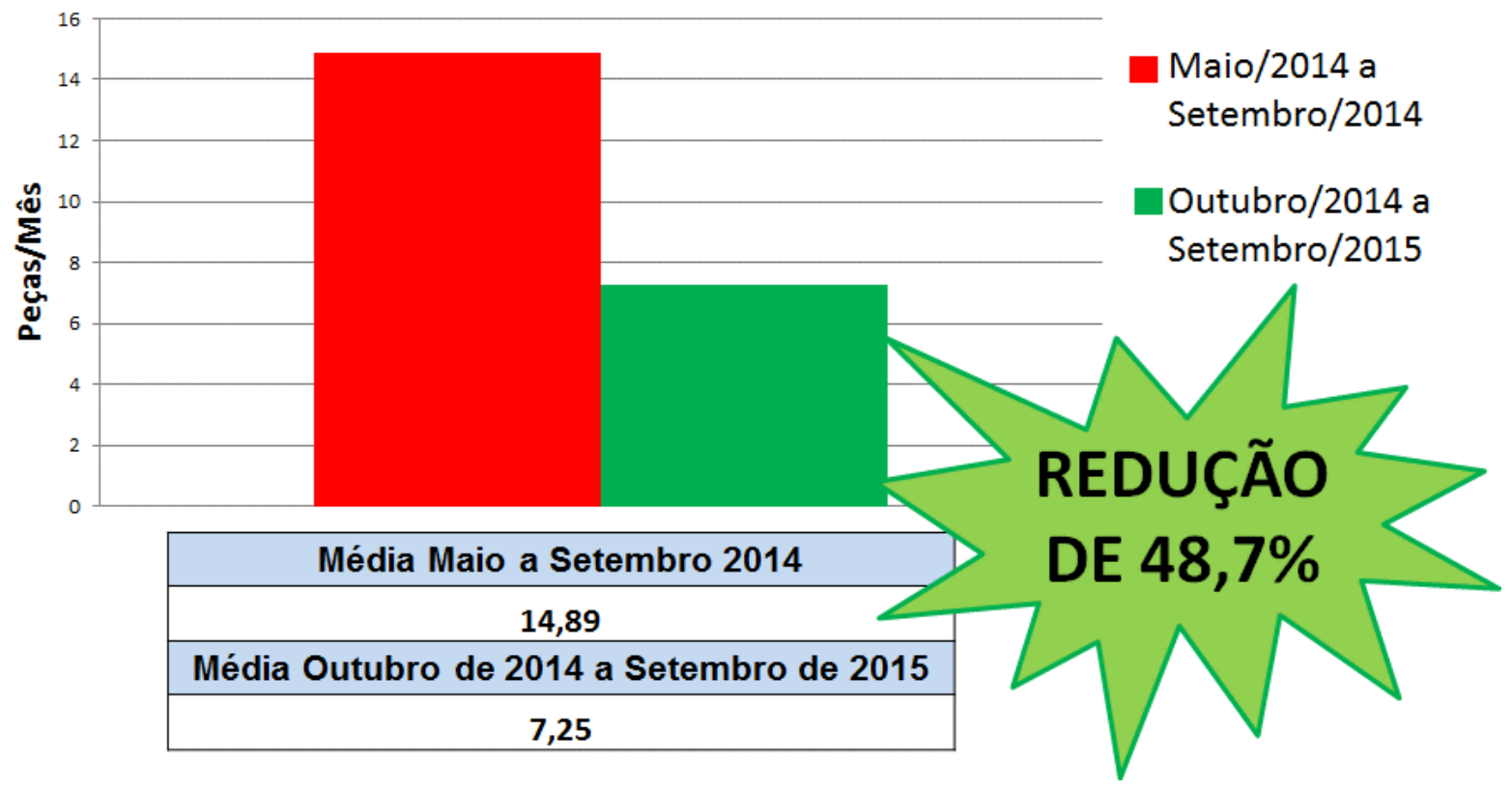

Figura 12 - Gráfico de Quebras 


\subsubsection{Redução do POWER ON}

\section{Média POWER ON}

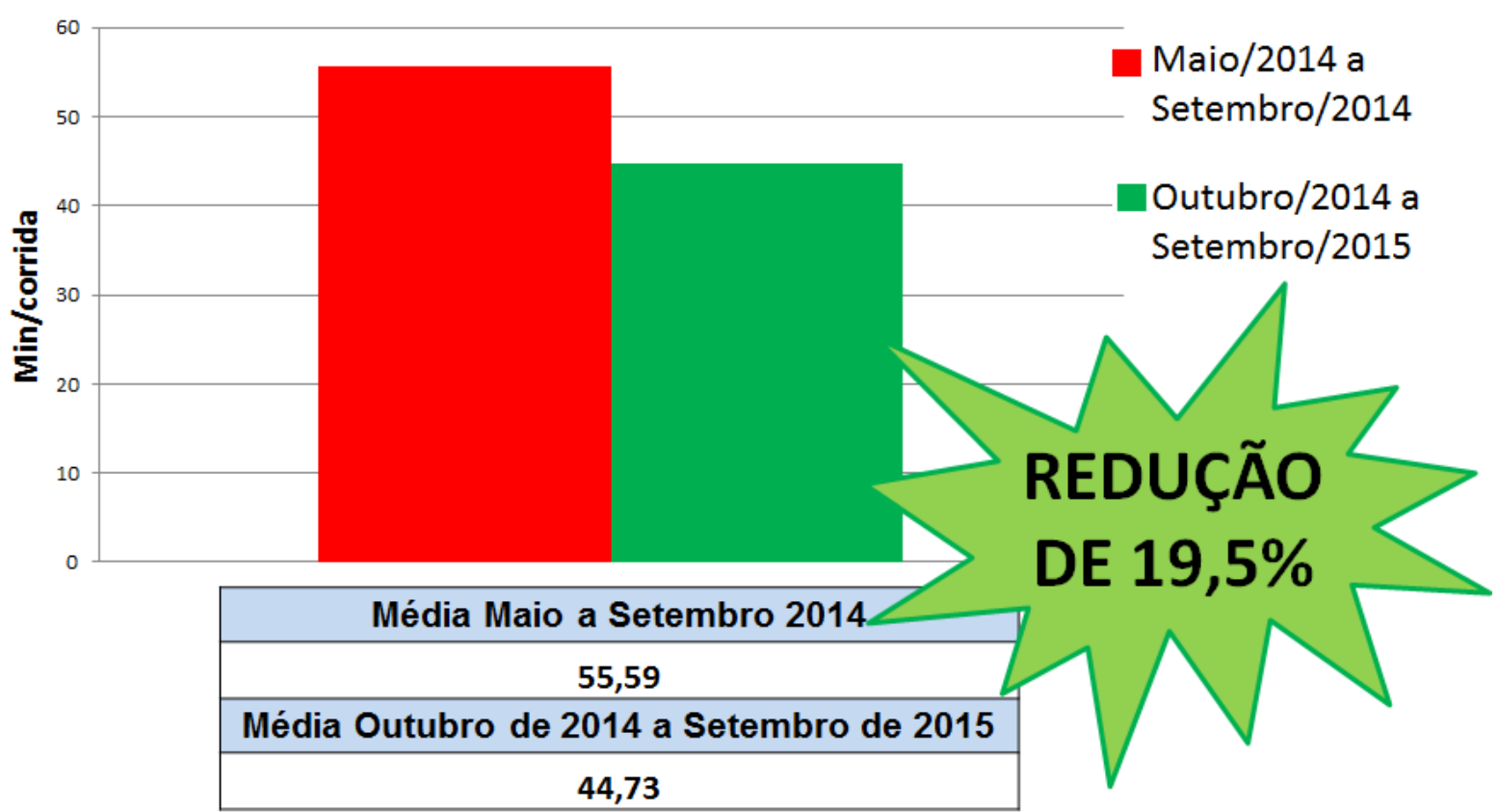

Fonte: ROLAP E PROCOM

Figura 13 - Redução do Power On

\subsubsection{Aumento da Produção}

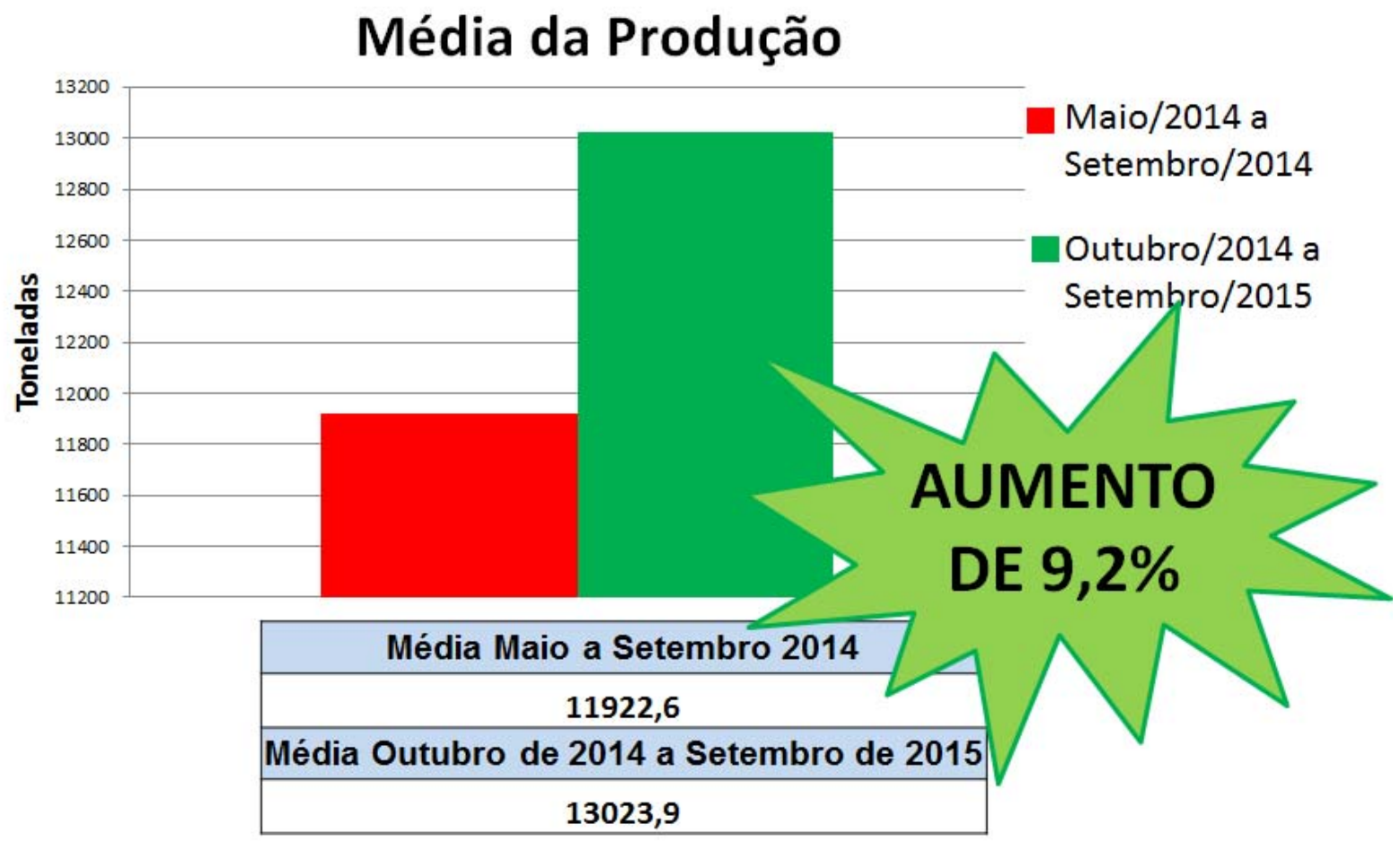

Fonte: ROLAP E PROCOM

Figura 14 - Media de Produção 


\subsubsection{Redução do tempo TAP-TO-TAP}

Média de Tempo de uma Corrida (TAP-TO-TAP)

\section{Antes [69 minutos]}

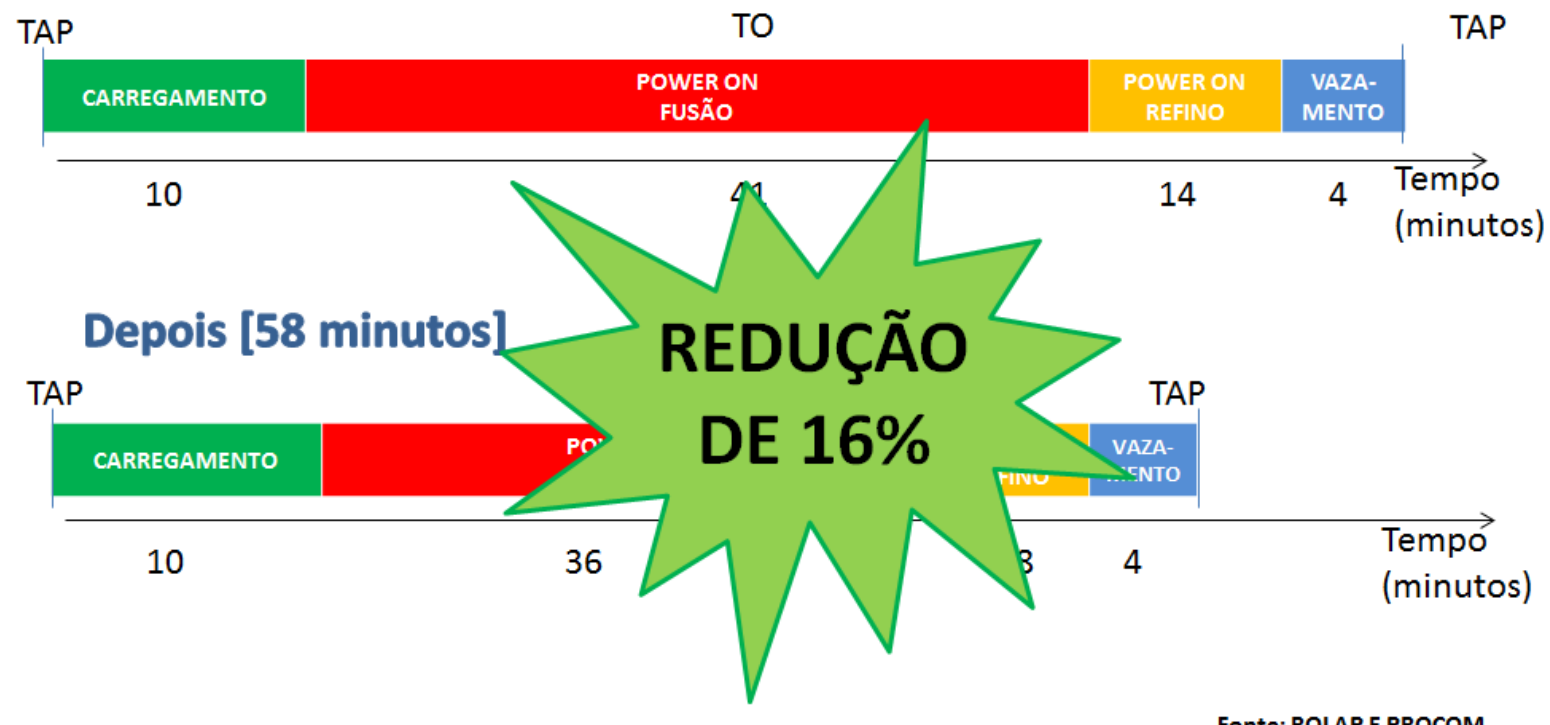

Figura 15 - tempo TAP TO TAP

\section{PADRONIZAÇÃO E TREINAMENTO}

5.1. Todos os documentos originais foram revisados e inseridos no sistema de documentação digital da empresa bem com a inclusão de novos documentos.

5.2. Todos os mantenedores e operadores do sistema de fusão a arco receberam treinamentos teóricos e práticos.

\section{CONCLUSÃO}

A expertise dos profissionais CSN agregou ao projeto original funcionalidades que permitiram aumentar a confiabilidade de um dos ativos mais importantes, disponibilizando análise de variáveis para otimização do processo reduzindo gastos com matéria prima e aumentando a competitividade do Negócio Aços Longos.

\section{AGRADECIMENTOS}

A todos os colaboradores que contribuíram para a realização deste projeto.

\section{REFERÊNCIAS}

1 EMT 053 - Processos Metalúrgicos. Assunto: Aciaria Elétrica - Prof. Renato Minelli Figueira

2 Manual de Operação 09/2014 de Aciaria Elétrica - Forconsult

3 Telas gráficas do Sistema SCADA Intouch do sistema implantado

4 Sistema de gerenciamento de dados de Processo da CSN - ROLAP e PROCOM 